ARTICLE

\title{
How to Combine Binary Collision Approximation and Multi-Body Potential for Molecular Dynamics
}

\author{
Seiki SAITO ${ }^{1, *}$, Arimichi TAKAYAMA ${ }^{2}$, Atsushi M. ITO2, \\ Takahiro KENMOTSU ${ }^{3}$ and Hiroaki NAKAMURA ${ }^{1,2}$ \\ ${ }^{1}$ Department of Energy Engineering and Science, Graduate School of Engineering, Nagoya University, \\ Furo-cho, Chikusa-ku, Nagoya, 464-8603, Japan. \\ ${ }^{2}$ Fundamental Physics Simulation Research Division, National Institute for Fusion Science, Toki, Gifu, 509-5292, Japan. \\ ${ }^{3}$ Faculty of Life and Medical Sciences, Doshisha University, Kyotanabe, Kyoto, 610-0394, Japan.
}

\begin{abstract}
Our group has been developing a hybrid simulation of the molecular dynamics (MD) and the binary collision approximation (BCA) simulation. One of the main problems of this hybridization model is that the multi-body potential suddenly appears at the moment when the simulation method switches from the BCA to the MD. This instantaneously emerged multi-body potential causes the acceleration or deceleration of atoms of the system. To solve this problem, the kinetic energy of atoms should be corrected to conserve the total energy in the system. This paper gives the solution. The hybrid simulation for hydrogen atom injection into a graphite material is executed in order to demonstrate the solution.
\end{abstract}

KEYWORDS: hybrid simulation, binary collision approximation, ACAT, molecular dynamics simulation, graphite, hydrogen, plasma-wall interaction

\section{Introduction}

To achieve steady-state operation of fusion plasmas, the understanding of the mechanism of chemical and physical interactions between plasmas and divertor plates is necessary. Our group has been investigating hydrogen-graphite interactions by mean of molecular dynamics (MD) simulation ${ }^{1-3}$ ) using modified Brenner's reactive empirical bond order (REBO) potential ${ }^{4,5)}$ which is modeled as a multi-body potential function depending on surrounding atoms. The MD simulation treats chemical reaction and the binding from surrounding atoms. However, the MD is not applicable for simulations of atoms in high energy because of the following three reasons: the MD simulation does not treat inelastic interactions by electron excitation. In general, the inelastic interactions cannot be ignored at high energy scattering; The MD simulation calculates the time evaluation by numerical integration. The time step has to be reduced to treat high-speed atoms; The Brenner's REBO potential is not adequate for high potential energy, because the Brenner's REBO potential was developed for hydrocarbons that can model intramolecular chemical bonding in a variety of small hydrocarbon molecules;

The physical sputtering is often calculated by binary collision approximation (BCA) simulation. In the BCA simulation, multi-body interactions in materials are approximate to consecutive two-body interactions between a projectile atom and the nearest neighbor atom. Each two-body interaction can be solved analytically, so the nu-

*Corresponding author, E-mail: saito.seiki@nifs.ac.jp merical integration is not necessary. The energy loss of inelastic interactions by electron excitation is also taken into account at each collision. We employ the Thomas-Fermi potential as the inter-atomic potential in our BCA code, which is named AC $\forall \mathrm{T}$ (atomic collision in any structured target) code. ${ }^{6}$ ) The computation time of the BCA is much shorter than that of the MD simulation. The BCA can treat large system of more than 1 million particles while the MD can treat less than 10 thousand particles system. However, BCA simulation is not appropriate for low energy collision because it ignores the interactions between surrounding atoms.

In order to expand the computable energy range and the size of simulation box, we have been developing a hybrid simulation of the MD and the BCA simulations. ${ }^{7)}$ The concept of hybridization of the MD and the BCA simulation is that the BCA simulation works when a kinetic energy of a projectile atom is higher than a threshold energy $E_{\text {th }}$, then the MD simulation starts when the kinetic energy becomes lower than the threshold energy $E_{\mathrm{th}}$. One of the main problems of this hybridization model is that the multi-body potential suddenly appears when the kinetic energy of the projectile atom becomes $E_{\mathrm{th}}$. This instantaneously emerged multi-body potential causes the acceleration or deceleration of atoms in the system.

In this paper, we overview the hybrid simulation model and the algorithms of the BCA and the MD simulation in Section II. Then we explain the energy correction method for the sake of the emerged multi-body potential energy when the simulation method switches from the BCA to the MD in Section III. Using the hybrid simulation code with the ener- 
gy correction method, we demonstrate and discuss the simulation results for hydrogen atom injection into a graphite material in Section IV. We summarize the paper in Section $\mathrm{V}$.

\section{Simulation Model}

\section{BCA Simulation Model}

A binary collision between a projectile and a target atom is calculated in the BCA simulation. The final position and velocity of the projectile and the target atom at each collision are obtained analytically in a two-body interatomic potential $V(r)$, where $r$ is the distance between the projectile and the target atom. In our BCA simulation code $\mathrm{AC} \forall \mathrm{T}$, the Moliere approximation to the Thomas-Fermi potential ${ }^{8)}$ is employed.

Figure 1 shows the trajectory of two particles interacting according to a conservative central repulsive force. The scattering angle in the center-of-mass system (CM-system) is

$$
\Theta^{\mathrm{CM}}=\pi-2 b \int_{r_{0}}^{\infty} \frac{1}{r^{2} g(r)} d r,
$$

where

$$
g(r)=\sqrt{1-\frac{b^{2}}{r^{2}}-\frac{V(r)}{E_{\mathrm{r}}}},
$$

$b$ is the impact parameter, $E_{\mathrm{r}}=E_{0} m_{1} /\left(m_{1}+m_{2}\right)$ is the relative kinetic energy, $E_{0}$ is the incident kinetic energy of the projectile, $r_{0}$ is the solution of $g(r)=0, m_{1}$ and $m_{2}$ are the mass of the projectile and the target atom, respectively. The trajectories of particles are approximated as the asymptotes of them in the laboratory system (L-system). So they consist of linkage of straight-line segments. The starting point of the projectile and the recoil atom after a collision is given by $\Delta x_{1}$ and $\Delta x_{2}$, which are the shifts from the initial position of the target atom shown in Fig. 1:

$$
\begin{aligned}
& \Delta x_{1}=\frac{2 \tau+(A-1) b \tan (\Theta / 2)}{1+A}, \\
& \Delta x_{2}=b \tan (\Theta / 2)-\Delta x_{1},
\end{aligned}
$$

where

$$
\tau=\sqrt{r_{0}^{2}-b^{2}}-\int_{r_{0}}^{\infty}\left\{\frac{1}{g(r)}-\frac{r}{\sqrt{r^{2}-b^{2}}}\right\} d r,
$$

and the mass ratio $A=m_{2} / m_{1}$.

In the collision process of the $\mathrm{AC} \forall \mathrm{T}$ simulation, the motion of target atoms which collide with a projectile depends on their received kinetic energy $E_{\mathrm{R}}$. When $E_{\mathrm{R}}$ is lower than the binding energy of target material, the target atom cannot move. When $E_{\mathrm{R}}$ is larger than the binding energy, the target atom recoils.

The AC $\forall \mathrm{T}$ code also evaluates the energy loss by electron excitation for each collision. For hydrogen, the four-parameter fitting formula ${ }^{9)}$ of the electronic stopping cross section which was originally proposed by Varelas and Biersack is employed.

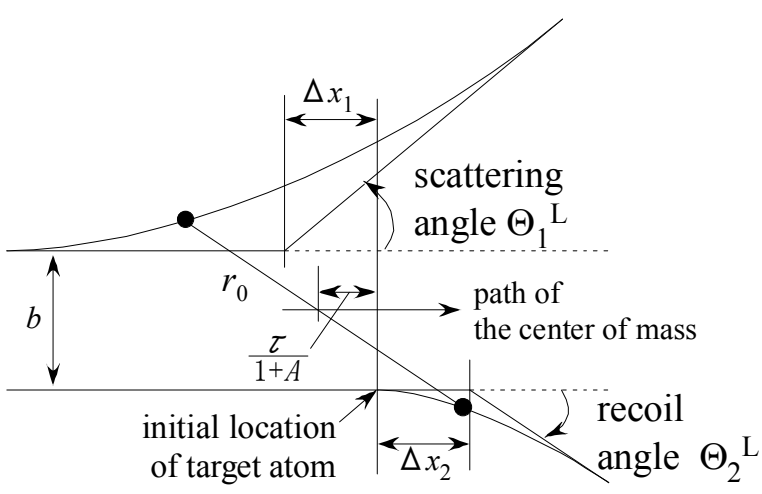

Fig. 1 The trajectory of two particles interacting according to a conservative central repulsive force in the laboratory system. The positions of the projectile and the target atom correspond to the apsis of the collision. ${ }^{12)}$

$$
S_{e}^{\mathrm{H}}(E)^{-1}=\left(S_{\mathrm{LOW}}^{\mathrm{H}}\right)^{-1}+\left(S_{\mathrm{HIGH}}^{\mathrm{H}}\right)^{-1},
$$

where

$$
\begin{aligned}
& S_{\mathrm{LOW}}^{\mathrm{H}}=A_{1} E^{0.45}, \\
& S_{\mathrm{HIGH}}^{\mathrm{H}}=\frac{A_{2}}{E} \ln \left[1+\frac{A_{3}}{E}+A_{4}\right],
\end{aligned}
$$

and four parameters $A_{1}, A_{2}, A_{3}, A_{4}$ are derived from fitting experimental data with the following theoretical expressions:

$$
\begin{aligned}
& S_{\mathrm{LOW}}=Z_{1}^{1 / 6} \times 8 \pi e^{2} a_{0} \frac{Z_{1} Z_{2}}{\left(Z_{1}^{2 / 3}+Z_{2}^{2 / 3}\right)^{3 / 2}} \times\left[\frac{v_{\mathrm{i}}}{v_{0}}\right], \\
& S_{\mathrm{HIGH}}=\frac{4 \pi e^{4} Z_{1}^{2} Z_{2}}{m v_{\mathrm{i}}^{2}} \times\left[\ln \frac{2 m v_{\mathrm{i}}^{2}}{I}+\ln \frac{1}{1-\beta}-\beta^{2}-\frac{C}{Z_{2}}\right],
\end{aligned}
$$

where $e$ and $m_{\mathrm{e}}$ are the charge and mass of an electron. $Z_{1}$ and $Z_{2}$ are the atomic numbers of a projectile and target atom, respectively. $v_{\mathrm{i}}$ is the relative velocity between projectile and target atom. $\beta$ is the ratio of $v_{\mathrm{i}}$ to the speed of light. $I$ is the mean excitation potential. $C$ is the shell-correction which is obtained by experimental data. ${ }^{9)} \quad a_{0}, v_{0}$ are the Bohr radius and velocity, respectively.

Equation (10) is the Bethe's stopping-power formula ${ }^{10)}$ which can be applied when Born approximation is applicable. Eq. (9) is the stopping-power formula derived by Lindhard ${ }^{11)}$ which can be applied for low speed ions where Born approximation is not applicable. Equations. (9) and (10) corresponds to $S^{\mathrm{H}}{ }_{\text {LOW }}$ and $S^{\mathrm{H}}{ }_{\mathrm{HIGH}}$, respectively.

\section{MD Simulation Model}

We execute a classical molecular dynamics simulation under the $N V E$ condition. The Hamiltonian in the MD simulation is defined by

$$
H=\sum_{i} \frac{p_{i}^{2}}{2 m_{i}}+U,
$$

where $p_{i}$ and $m_{i}$ are the momentum and the mass of the $i$-th atom, respectively. $U$ is a total potential energy. In our MD 
simulation, the modified REBO potential, which is widely used in MD simulation of carbon systems, is employed for the potential $U$. A carbon atom has four (or fewer) covalent bonds, which result from four valence electrons. The potential function depends strongly on the bonding state of carbon atom. The REBO potential is defined as:

$$
\begin{aligned}
& U \equiv \sum_{i, j>i} U_{i j}, \\
& U_{i j} \equiv V_{[i j]}^{\mathrm{R}}\left(r_{i j}\right)-\bar{b}_{i j}\left(\{r\},\left\{\theta^{\mathrm{B}}\right\},\left\{\theta^{\mathrm{DH}}\right\}\right) V_{[i j]}^{\mathrm{A}}\left(r_{i j}\right),
\end{aligned}
$$

where $r_{i j}$ is the distance between the $i$-th and the $j$-th atoms. The functions $V^{\mathrm{R}}{ }_{[i j]}$ and $V^{\mathrm{A}}{ }_{[i j]}$ represent repulsion and attraction, respectively. The function $\bar{b}_{i j}$ generates a multi-body force. The parameters $\{r\},\left\{\theta^{\mathrm{B}}\right\}$, and $\left\{\theta^{\mathrm{DH}}\right\}$ denote all sets of $r_{i j}, \theta_{j i k}^{\mathrm{B}}$, and $\theta^{\mathrm{DH}}{ }_{k i j}$, respectively. The bond angle $\theta^{\mathrm{B}}{ }_{j i k}$ is the angle between the vector from the $i$-th atom to the $j$-th atom and the vector from the $i$-th atom to the $k$-th atom. The dihedral angle $\theta_{\text {kijl }}^{\mathrm{DH}}$ is the angle between the plane passing through the $k$-th, $i$-th, and $j$-th atoms and the plane passing through the $i$-th, $j$-th, and $l$-th atoms (for details of the modified Brenner's REBO potential, see References 2 and 13. The second-order symplectic integration ${ }^{14)}$ is used to execute the time integration of the equation of motion, where the time step is set to $5 \times 10^{-18} \mathrm{~s}$.

\section{BCA-MD Hybrid Simulation Model}

The BCA simulation has a typical advantage for availability of collision with high kinetic energy. The MD simulation has an advantage for low energy collision. Therefore, it is possible to extend the treatable energy range by connecting these two models. The hybrid simulation treats both physical and chemical reaction simultaneously.

Figure 2 shows the concept of the hybrid simulation on the example of hydrogen injection into a graphite material. The injection kinetic energy of the hydrogen atom is set to higher kinetic energy than $E_{\mathrm{th}}$. While the kinetic energy of the hydrogen atom is higher than $E_{\mathrm{th}}$, trajectories of the hydrogen atom and the surrounding carbon atoms are calculated by the BCA simulation. The hydrogen atom dissipates its kinetic energy by interacting with carbon atoms in graphite and then the kinetic energy becomes lower than $E_{\mathrm{th}}$. At that moment, the MD simulation starts to calculate the motions of the hydrogen atom and the surrounding carbon atoms in a box which is called MD simulation box.

In the collision process of the BCA simulation, some carbon atoms recoil. The motion of recoil carbon atoms is solved in the same way that the motion of the incident hydrogen is solved in the BCA and the MD simulation.

\section{Energy Correction Method}

\section{Concept}

We explain the energy correction method for the sake of the emerged multi-body potential energy when the simulation method switches from the BCA to the MD.

In the BCA simulation, the kinetic energies of projectile and recoil atoms after a collision are given under the condition the inter potential energy $V(r)$ is equal to zero. In the

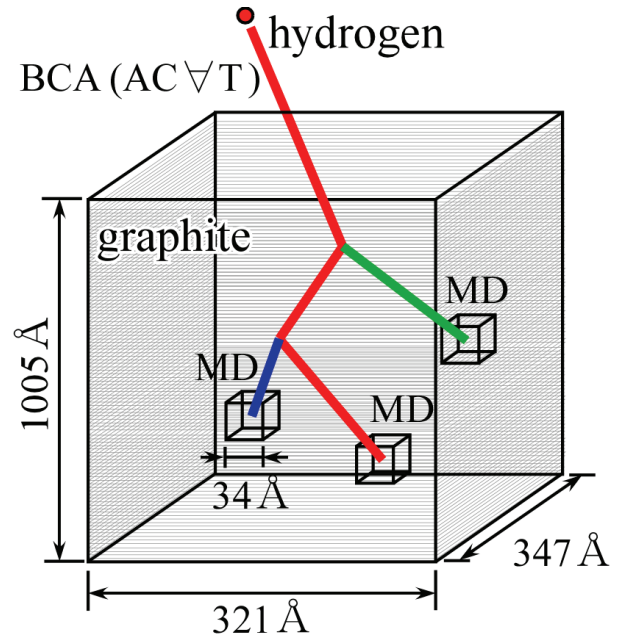

Fig. 2 Schematic diagram of the BCA-MD hybrid simulation of hydrogen injection into a graphite ${ }^{7)}$.

MD simulation, the potential energy depends on the changes of the position of atoms in materials. The difference of potential $\Delta U_{\text {tot }}$ between the initial state and the final state of BCA should be subtracted from the final kinetic energy of BCA simulation before starting the MD simulation. Therefore, we execute the energy correction right after the BCA simulation finished.

\section{Definition of Notation}

A simulation that an incident atom is injected into a target material is considered. The incident atom collides with target atoms, and then some of target atoms recoil. The recoil atoms derive cascade process by colliding with other target atoms. Figure 3(a) shows the diagram of a collision process in BCA simulation.

We divide the collision process into collision-sets. A collision-set include one recoil atom (see Fig. 3(a)). The collision-set is numbered in the order of the calculation. In the $\mathrm{AC} \forall \mathrm{T}$ simulation, the trajectory of a recoil atom is calculated and then that of projectile is calculated. Parameters $s$ represents a number of collision-set. $s=1$ and $s=s_{f}$ represent the first and final collision-set, respectively.

As shown in Fig. 3(b), parameters $P(s)$ and $R(s)$ are used for the indexes of projectile and recoil atom in $s$-th collision-set. The variable $s$ for $P(s)$ and $R(s)$ is omitted as $P$ and $R$ when it is clear that the descriptions of $s$-th collision-set.

The position and kinetic energy of the $i$-th atom at the initial state in the $s$-th collision-set are written as $\boldsymbol{r}_{i}(s)$ and $\varepsilon_{i}(s)$, respectively. Those of the final state are written as $\boldsymbol{r}_{i}{ }^{\prime}(s)$ and $\varepsilon_{i}^{\prime}(s)$. We use $N$ for total number of atoms.

As shown in Fig. 3(c), the corrected kinetic energy of $\varepsilon_{i}$ is written as $\bar{\varepsilon}_{i}$. The corrected kinetic energy of the final state of the final collision-set $\bar{\varepsilon}_{i}^{\prime}\left(s_{f}\right)$ is used for the MD simulation.

In the initial state, only an incident atom has kinetic energy $\varepsilon_{P(1)}(1)$, and kinetic energies of other atoms $\varepsilon_{i}(1)$ $(i \neq P(1))$ are equal to zero. We note here that the initial ki- 


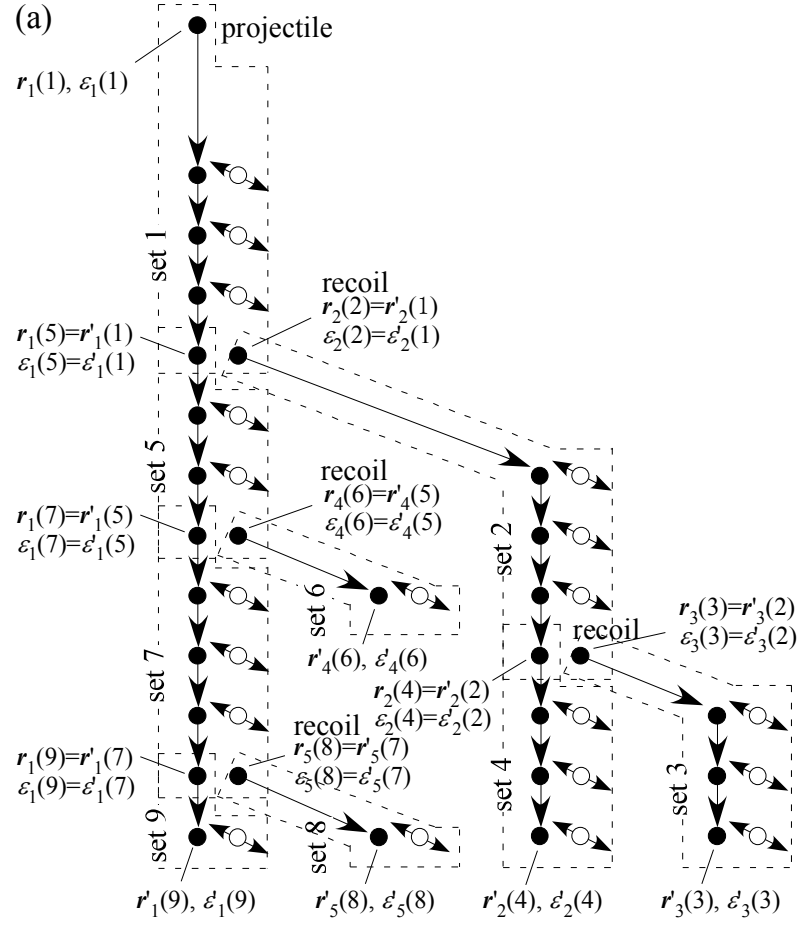

$\boldsymbol{r}_{P}(s), \varepsilon_{P}(s)$

(b)

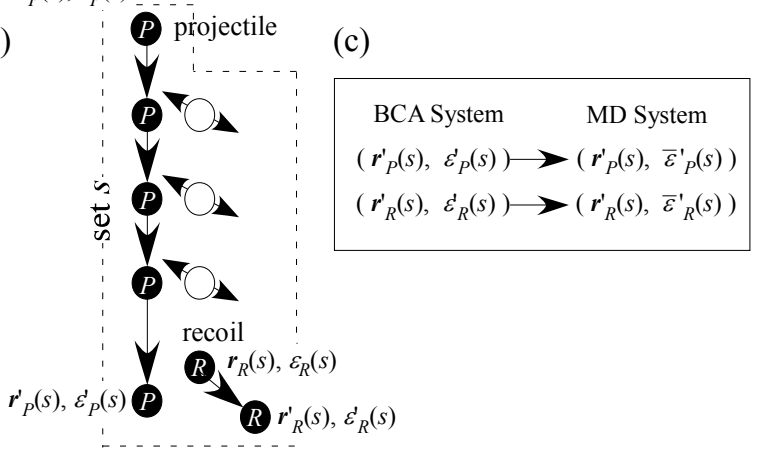

Fig. 3 (a) Diagram of a collision process in the BCA simulation. The white circles represent the target atoms which do not recoil. Each set enclosed by a dashed line represents a collision set. (b) The definition of notation for the $s$-th collision-set. (c) The concept of the energy correction. The kinetic energy calculated by the BCA is recalculated for the MD simulation.

netic energy of incident atom in the MD system $\overline{\mathcal{E}}_{P(1)}(1)$ is equal to $\varepsilon_{P(1)}(1)$ because the incident atom comes from infinite distance.

\section{Algorithm}

The final kinetic energy of moving atoms in the BCA and the initial kinetic energy of moving atoms in the MD satisfy the following relation:

$$
\begin{aligned}
& \sum_{i=1}^{N} \varepsilon_{i}^{\prime}\left(s_{f}\right)-\sum_{i=1}^{N} \varepsilon_{i}(1)= \sum_{i=1}^{N} \bar{\varepsilon}_{i}^{\prime}\left(s_{f}\right)-\sum_{i=1}^{N} \bar{\varepsilon}_{i}(1)+\Delta U_{\mathrm{tot}}, \\
& \Delta U_{\mathrm{tot}} \equiv U\left(\boldsymbol{r}_{1}^{\prime}\left(s_{f}\right), \boldsymbol{r}_{2}^{\prime}\left(s_{f}\right), \cdots, \boldsymbol{r}_{N}^{\prime}\left(s_{f}\right)\right) \\
&-U\left(\boldsymbol{r}_{1}(1), \boldsymbol{r}_{2}(1), \cdots, \boldsymbol{r}_{N}(1)\right),
\end{aligned}
$$

where $\Delta U_{\text {tot }}$ is the change of potential energy from initial to final state. Because $\bar{\varepsilon}_{i}(1)$ is equal to $\varepsilon_{i}(1)$, Eq. (14) also expresses the conservation of the total energy of both the BCA and the MD system at the final state of the collision process.

The material becomes the final state $\left(\boldsymbol{r}_{1}{ }_{1}\left(s_{f}\right), \boldsymbol{r}_{2}{ }_{2}\left(s_{f}\right), \ldots\right.$, $\left.\boldsymbol{r}_{N}\left(s_{f}\right)\right)$ via binary collisions. The change of potential energy is caused at each collision when the target atom recoils. Therefore, the difference of potential energy $\Delta U_{\text {tot }}$ can be described as follows:

$$
\begin{aligned}
& \Delta U_{\mathrm{tot}}=\sum_{s} \Delta U(s), \\
& \Delta U(s) \equiv U\left(\boldsymbol{r}_{1}(s), \boldsymbol{r}_{2}(s), \cdots, \boldsymbol{r}_{P}^{\prime}(s), \ldots, \boldsymbol{r}_{R}^{\prime}(s), \ldots, \boldsymbol{r}_{N}(s)\right) \\
& \quad-U\left(\boldsymbol{r}_{1}(s), \boldsymbol{r}_{2}(s), \cdots, \boldsymbol{r}_{P}(s), \ldots, \boldsymbol{r}_{R}(s), \ldots, \boldsymbol{r}_{N}(s)\right) .
\end{aligned}
$$

The energy correction is executed under Eq. (14) that is the condition of the conservation of the total energy of both the BCA and the MD system at the final state of the collision process. To conserve the total energy at the final state, the total energy loss in each collision-set, that is energy loss of electron excitation and of kinetic energy transferred to the target atoms which do not recoil should be equal in both the BCA and the MD system. Therefore $\bar{\varepsilon}_{P}^{\prime}(s)$ and $\bar{\varepsilon}_{R}^{\prime}(s)$ satisfy the following equation:

$$
\begin{aligned}
& \varepsilon_{P}^{\prime}(s)+\varepsilon_{R}^{\prime}(s)-\varepsilon_{P}(s) \\
& \quad=\bar{\varepsilon}_{P}^{\prime}(s)+\bar{\varepsilon}_{R}^{\prime}(s)-\bar{\varepsilon}_{P}(s)+\Delta U(s) .
\end{aligned}
$$

We note here that Eq. (14) can be derived by adding the Eq. (18) for all collision-sets. We define the difference of kinetic energy between the BCA and the MD system as follows:

$$
\begin{aligned}
& \Delta \varepsilon_{P}(s) \equiv \varepsilon_{P}(s)-\bar{\varepsilon}_{P}(s), \\
& \Delta \varepsilon_{P}^{\prime}(s) \equiv \varepsilon_{P}^{\prime}(s)-\bar{\varepsilon}_{P}^{\prime}(s), \\
& \Delta \varepsilon_{R}^{\prime}(s) \equiv \varepsilon_{R}^{\prime}(s)-\bar{\varepsilon}_{R}^{\prime}(s) .
\end{aligned}
$$

Then, Eq. (18) becomes

$$
\Delta \varepsilon_{P}^{\prime}(s)+\Delta \varepsilon_{R}^{\prime}(s)=\Delta U(s)+\Delta \varepsilon_{P}(s)
$$

We assume $\Delta \varepsilon_{P}^{\prime}(s)$ and $\Delta \varepsilon_{R}^{\prime}(s)$ can be written as follows:

$$
\begin{aligned}
& \Delta \varepsilon_{P}^{\prime}(s)=\frac{\Delta U_{P}(s)}{\Delta U_{P}(s)+\Delta U_{R}(s)} \Delta U(s)+\Delta \varepsilon_{P}(s), \\
& \Delta \varepsilon_{R}^{\prime}(s)=\frac{\Delta U_{R}(s)}{\Delta U_{P}(s)+\Delta U_{R}(s)} \Delta U(s)
\end{aligned}
$$

where $\Delta U_{P}(s)$ or $\Delta U_{R}(s)$ is defined as follows:

$$
\begin{aligned}
\Delta U_{P}(s) \equiv & U\left(\boldsymbol{r}_{1}(s), \boldsymbol{r}_{2}(s), \cdots, \boldsymbol{r}_{P}^{\prime}(s), \ldots, \boldsymbol{r}_{R}(s), \ldots, \boldsymbol{r}_{N}(s)\right) \\
& -U\left(\boldsymbol{r}_{1}(s), \boldsymbol{r}_{2}(s), \cdots, \boldsymbol{r}_{P}(s), \ldots, \boldsymbol{r}_{R}(s), \ldots, \boldsymbol{r}_{N}(s)\right), \\
\Delta U_{R}(s) \equiv & U\left(\boldsymbol{r}_{1}(s), \boldsymbol{r}_{2}(s), \cdots, \boldsymbol{r}_{P}(s), \ldots, \boldsymbol{r}_{R}^{\prime}(s), \ldots, \boldsymbol{r}_{N}(s)\right) \\
& -U\left(\boldsymbol{r}_{1}(s), \boldsymbol{r}_{2}(s), \cdots, \boldsymbol{r}_{P}(s), \ldots, \boldsymbol{r}_{R}(s), \ldots, \boldsymbol{r}_{N}(s)\right) .
\end{aligned}
$$

$\Delta \varepsilon_{P}(s)$ is obtained from the previous collision-set $s$. of 
(a)

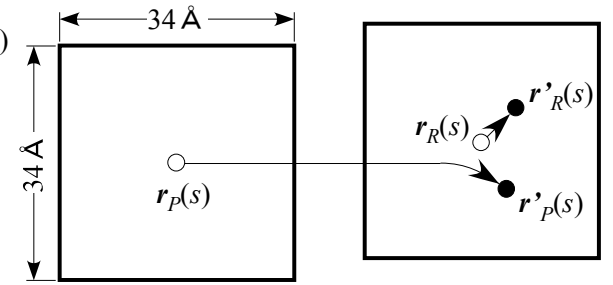

(b)

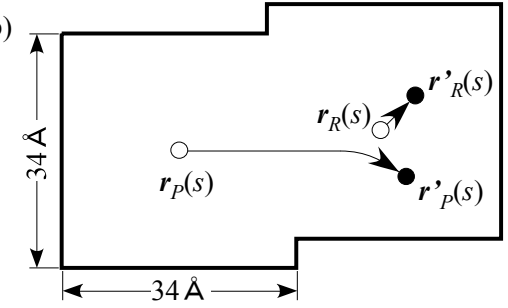

Fig. 4 (a) Two boxes whose centers are $\boldsymbol{r}_{P}(s)$ and $\boldsymbol{r}_{R}(s)$. The potential difference $\Delta U(s)$ for $s$-th collision set are calculated for the atoms in these boxes. (b) In the case that two boxes are combined as one domain because they are close.

projectile atom. (When $s^{=4,} s_{-}=2$ in the case of Fig. 3(a).)

$$
\begin{aligned}
& \Delta \varepsilon_{P}(1)=0, \\
& \Delta \varepsilon_{P}(s)=\left\{\begin{array}{ll}
\Delta \varepsilon_{P}^{\prime}\left(s_{-}\right) & \text {for } P(s)=P\left(s_{-}\right) \\
\Delta \varepsilon_{R}^{\prime}\left(s_{-}\right) & \text {for } P(s)=R\left(s_{-}\right)
\end{array} .\right.
\end{aligned}
$$

By solving $\Delta \varepsilon_{P}^{\prime}(s)$ and $\Delta \varepsilon_{R}^{\prime}(s)$ sequentially from the initial collision-set $s=1$, the final kinetic energies in the MD simulation $\bar{\varepsilon}_{i}^{\prime}\left(s_{f}\right),(i=1,2, \ldots, N)$ are obtained.

\section{Computational Method}

To obtain the final kinetic energies in the MD system $\bar{\varepsilon}_{i}^{\prime}\left(s_{f}\right)$, the potential difference $\Delta U(s)$ for each collision-set is required. The total number of atoms is more than 1 million. Therefore, it is not possible to calculate the potential function for all atoms in the system because of long computation time. Thus, we pick up approximately 4,000 atoms in a cubic box whose side is $34 \AA$ long. The bond energy between the $i$-th and the $j$-th atom $U_{i j}$ defined by Eq. (13) depends on the bonding state of the bonds which connecting in a distance of two bonds. When the $i$-th atom moves from $\boldsymbol{r}_{i}(s)$ to the $\boldsymbol{r}_{i}(s)$, the binding energies for the bonds whose distance is more than $34 \AA$ from $\boldsymbol{r}_{i}(s)$ and $\boldsymbol{r}_{i}(s)$ are not changed. Therefore, as shown in Fig. 4(a), the potential change for the $s$-th collision-set $\Delta U(s)$ can be calculated as follows:

$$
\begin{aligned}
& \Delta U(s)= \\
& U^{\mathrm{BOX}}\left(\boldsymbol{r}_{P}(s) ; \boldsymbol{r}_{P}^{\prime}(s), \boldsymbol{r}_{R}^{\prime}(s)\right)-U^{\mathrm{BOX}}\left(\boldsymbol{r}_{P}(s) ; \boldsymbol{r}_{P}(s), \boldsymbol{r}_{R}(s)\right) \\
& +U^{\mathrm{BOX}}\left(\boldsymbol{r}_{R}(s) ; \boldsymbol{r}_{P}^{\prime}(s), \boldsymbol{r}_{R}^{\prime}(s)\right)-U^{\mathrm{BOX}}\left(\boldsymbol{r}_{R}(s) ; \boldsymbol{r}_{P}(s), \boldsymbol{r}_{R}(s)\right),
\end{aligned}
$$

where $U^{\mathrm{BOX}}\left(\boldsymbol{r}_{\mathrm{C}} ; \boldsymbol{r}_{P}, \boldsymbol{r}_{R}\right)$ is the total potential energy of atoms in the box whose center is located at $\boldsymbol{r}_{\mathrm{C}}$ in the case that projectile and recoil atom are located at $\boldsymbol{r}_{P}$ and $\boldsymbol{r}_{R}$, respectively. When the two boxes overlap, the binding energies of the atoms in the boxes are not independent. In this case, the two

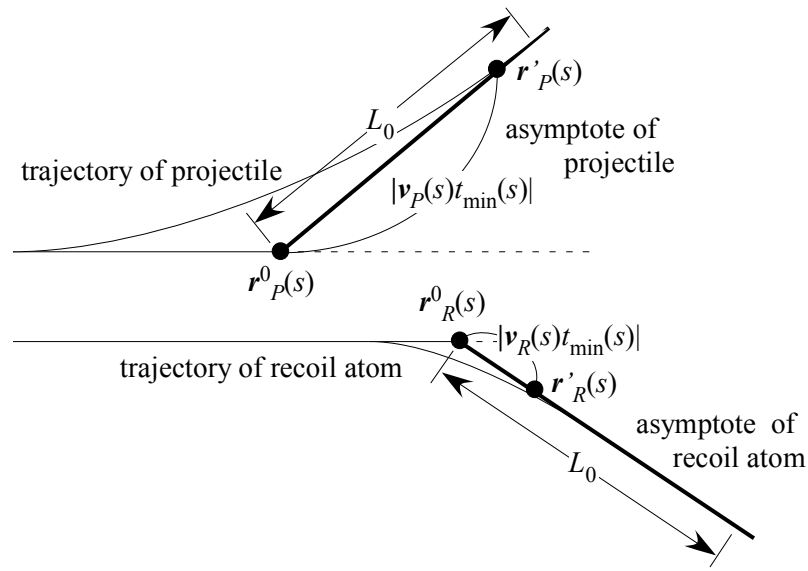

Fig. 5 The asymptotes of the projectile and the recoil atom after a collision with their starting point $\boldsymbol{r}_{P}^{0}$ and $\boldsymbol{r}_{R}^{0}$.

boxes are combined as one domain to calculate $\Delta U(s)$ as shown in Fig. 4(b).

The BCA simulation approximates the trajectories of projectile and recoil atoms to their asymptotes. Therefore, the position of projectile and recoil atom $\boldsymbol{r}_{P}{ }_{P}(s)$ and $\boldsymbol{r}_{R}{ }_{R}(s)$ can be set arbitrarily on their asymptotes. When $\boldsymbol{r}_{P}^{\prime}(s)$ and $\boldsymbol{r}_{R}{ }_{R}(s)$ are set in a short distance, the potential change $\Delta U(s)$ becomes large and $\Delta \varepsilon_{P}^{\prime}(s)$ and $\Delta \varepsilon_{R}^{\prime}(s)$ become larger than their original kinetic energies. When $\boldsymbol{r}_{P}{ }_{P}(s)$ and $\boldsymbol{r}_{R}(s)$ are set far away from their starting points of asymptotes $\boldsymbol{r}_{P}^{0}$ and $\boldsymbol{r}_{R}^{0}$ as shown in Fig. 5, collisions with neighbor atoms are neglected. To solve this problem, $\boldsymbol{r}_{P}{ }_{P}(s)$ and $\boldsymbol{r}_{R}{ }_{R}(s)$ are set at the position where $\Delta U(s)$ becomes minimum on their asymptotes in a distance of carbon bond length $L_{0}=1.45 \AA$ from their starting points $\boldsymbol{r}_{P}^{0}$ and $\boldsymbol{r}_{R}^{0}$. Therefore $\boldsymbol{r}_{P}{ }_{P}(s)$ and $\boldsymbol{r}_{R}{ }_{R}(s)$ are decided as follows:

$$
\begin{aligned}
& \boldsymbol{r}_{P}^{\prime}(j)=\boldsymbol{r}_{R}^{0}(s)+\boldsymbol{v}_{P}(s) t_{\text {min }}(s), \\
& \boldsymbol{r}_{R}^{\prime}(s)=\boldsymbol{r}_{R}^{0}(s)+\boldsymbol{v}_{R}(s) t_{\text {min }}(s),
\end{aligned}
$$

where, $\boldsymbol{v}_{\mathrm{P}}(s)$ and $\boldsymbol{v}_{\mathrm{R}}(s)$ are the velocity of projectile and recoil atoms after collision, respectively. $t_{\min }(s)$ is defined as follows:

$$
t_{\text {min }}= \begin{cases}\left|\boldsymbol{r}_{P}^{\prime}(s)-\boldsymbol{r}_{P}^{0}(s)\right|\left|\boldsymbol{v}_{P}(s)\right| & \text { for } \boldsymbol{v}_{R}(s)\left|\leq \boldsymbol{v}_{P}(s)\right| \\ \boldsymbol{r}_{R}^{\prime}(s)-\boldsymbol{r}_{R}^{0}(s)\left|/ \boldsymbol{v}_{R}(s)\right| & \text { for } \boldsymbol{v}_{R}(s)\left|>\boldsymbol{v}_{P}(s)\right|\end{cases}
$$

\section{Numerical Demonstration}

We demonstrate an AC $\forall \mathrm{T}-\mathrm{MD}$ hybrid simulation for a hydrogen atom injection into an ideal graphite material which is $321 \AA$ long, $347 \AA$ wide and $335 \AA$ deep. The graphite consists of 100 sheets of graphene. Periodic boundary conditions are imposed on the horizontal direction. The initial temperature of the graphite is set to zero Kelvin. The hydrogen atom with the kinetic energy of $1 \mathrm{keV}$ is injected vertically into the graphite. The threshold kinetic energy $E_{\text {th }}$ is set to $200 \mathrm{eV}$. ${ }^{7)}$

Figure 6 shows two trajectories of incident hydrogen in the same initial condition. One of them is calculated by ap- 
plying the energy correction. The other one is calculated without the energy correction. Both trajectories obtained by the BCA simulation are the same. After the simulation method switches to the MD, these trajectories disagree because the kinetic energy of hydrogen is corrected.

Figure 7(a) shows the evolution of kinetic energy of incident hydrogen atom $\varepsilon_{\mathrm{H}}$, total kinetic energy of carbon atoms $\varepsilon_{\mathrm{C}}$ and total potential energy $U$ when energy correction is applied. $\varepsilon_{\mathrm{C}}$ includes the kinetic energy of the carbon atoms which receive lower kinetic energy than its binding energy at collisions. In the BCA simulation, $\varepsilon_{\mathrm{H}}$ decreases and $\varepsilon_{\mathrm{C}}$ increases at each collision. A recoil atom is generated during the BCA simulation in the sample trajectory. At this moment, $\varepsilon_{\mathrm{C}}$ increases a lot. The difference between incident energy $1 \mathrm{keV}$ and total energy $\varepsilon_{\mathrm{H}}+\varepsilon_{\mathrm{C}}$ is the energy loss by electron excitation. When the kinetic energy of the projectile atom reaches $E_{\mathrm{th}}=200 \mathrm{eV}$, the simulation method switches to the MD simulation, the multi-body potential is suddenly appeared.

Figure 7(b) shows the close-up of Fig. 7(a) at the switching point. The emerged potential energy at the switching point is $15.38 \mathrm{eV}$. Therefore, the total kinetic energy $\varepsilon_{\mathrm{H}}+\varepsilon_{\mathrm{C}}$ is reduced by $15.38 \mathrm{eV}$ for the energy correction. As the result, the total energy $\varepsilon_{\mathrm{H}}+\varepsilon_{\mathrm{C}}+U$ is conserved. Figure 7 (c) shows the evolution at the switching point in the case that energy correction is not applied. It is clearly seen that the total energy is not conserved at the switching point.

In the MD simulation, $\varepsilon_{\mathrm{H}}$ ripples. This is because the $U$ rises when the hydrogen atom penetrates a sheet of graphene and it falls when the hydrogen atom is located at the interlayer. While the hydrogen atom is penetrating the graphene, the carbon atoms of the graphene receives kinetic energy from the hydrogen atom and they move from their stability point of the potential. Therefore, every time the hydrogen atom penetrates the graphene, $\varepsilon_{\mathrm{C}}$ and $U$ increase.

\section{Summary and Future Work}

The energy correction method for the sake of the emerged multi-body potential energy when the simulation method switches from the BCA to the MD was explained. The kinetic energy of projectile and recoil atom is corrected to conserve the total energy loss in each collision set in both the BCA and MD systems.

AC $\forall \mathrm{T}-\mathrm{MD}$ hybrid simulation for a hydrogen atom injection into an ideal graphite material was performed with the energy correction. The total energy at the moment when the simulation method switches to the MD is conserved.

By applying the energy correction, it is expected that the path length to stopping of ions in materials would be shortened. We are planning to confirm that by taking statistics.

Another method for the energy correction is considerable. For example, in the case $\Delta \varepsilon_{P}\left(s_{f}\right)$ is small enough to be negligible, the correction can be calculated by taking only the final collision into account. In this case, the correction is analytically solved by assuming the momentum and angular momentum conservation for the final collision. We are planning to compare this method with the method described in this paper.

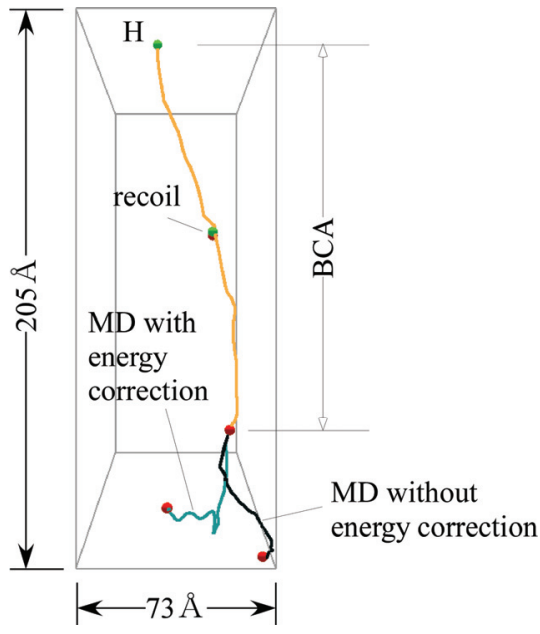

Fig. 6 Two trajectories of incident hydrogen in the same initial condition. The blue and black line is the trajectory calculated with the energy correction and without the energy correction, respectively. The yellow line is the trajectory calculated by BCA simulation.
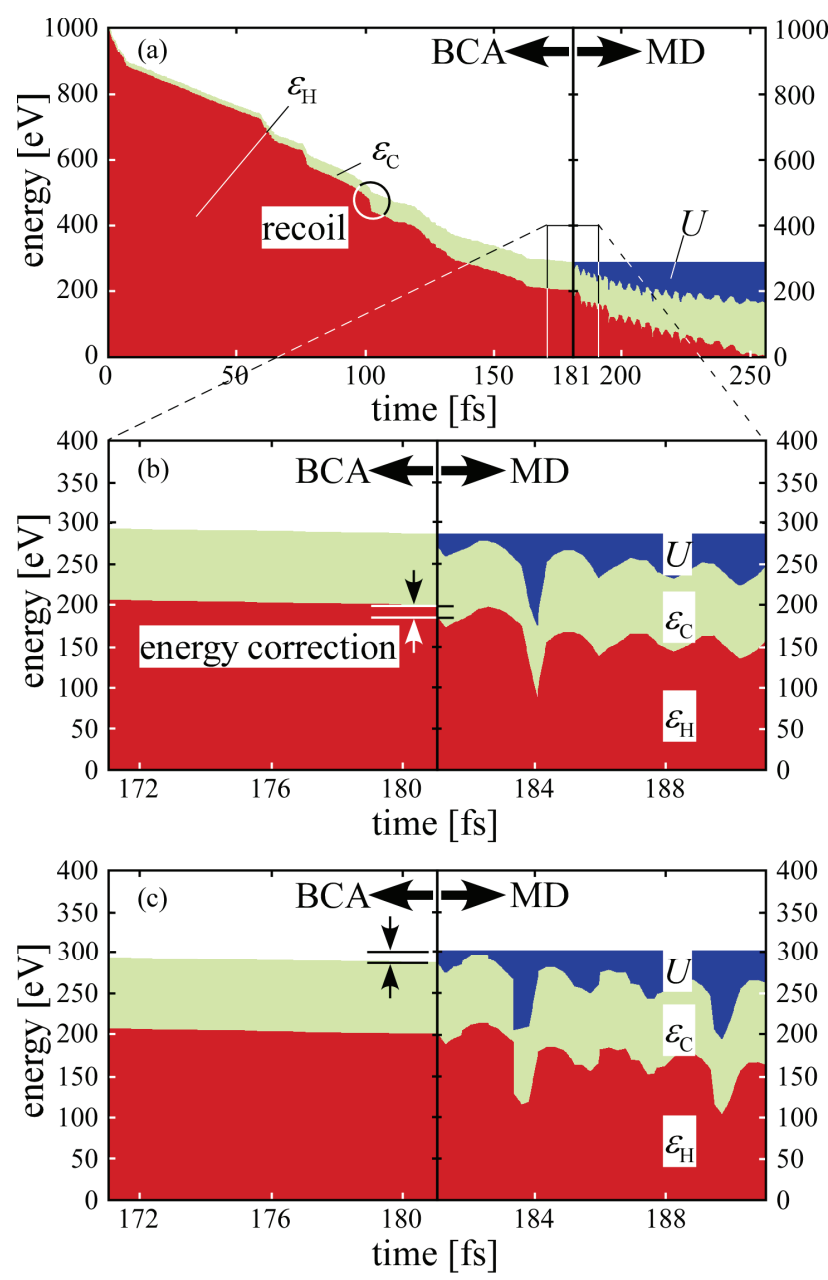

Fig. 7 (a) The evolution of kinetic energy of incident hydrogen atom $\varepsilon_{\mathrm{H}}$ (red), total kinetic energy of carbon atoms $\varepsilon_{\mathrm{C}}$ (green) and total potential energy $U$ (blue) when energy correction is applied. (b) The close-up of (a) at the switching point. (c) The evolution when the energy correction is not applied. 


\section{Acknowledgement}

This work is supported by the National Institutes of Natural Sciences' undertaking Forming Bases for Interdisciplinary and International Research through Cooperation Across Fields of Study and Collaborative Research Programs (NIFS09KEIN0091 and NIFS10KEIN0161) and a Grants-in-Aid for Scientific Research (No. 19055005) from the Ministry of Education, Culture, Sports, Science and Technology, Japan. This work is performed with the support and under the auspices of the NIFS Collaboration Research programs (NIFS09KTAN008 and NIFS10KTAS005).

\section{References}

1) A. Ito, H. Nakamura, "Molecular dynamics simulation of collisions between hydrogen and graphite," J. Plasma Phys., 72, 805-808 (2006).

2) A. Ito, H. Nakamura, A. Takayama, "Molecular Dynamics Simulation of the Chemical Interaction between Hydrogen Atom and Graphene," J. Phys. Soc. Jpn., 77, 114602 (2008).

3) S. Saito, A. Ito, H. Nakamura, "Molecular Dynamics Simulation of the Incident Angle Dependence of Reactions between Graphene and Hydrogen Atom", Plasma Fusion Res., Special Issue, 5, S2076 (2010).

4) D. W. Brenner, O. A. Shenderova, J. A. Harrison, S. J. Stuart, B. $\mathrm{Ni}$, S. B. Sinnott, "A second-generation reactive empirical bond order (REBO) potential energy expression for hydrocarbons," $J$. Phys. Condens. Matter, 14, 783-802 (2002).
5) A. Ito, H. Nakamura, "Molecular Dynamics Simulation of Bombardment of Hydrogen Atoms on Graphite Surface," Commun. Comput. Phys., 4, 592-610 (2008).

6) A. Takayama, S. Saito, A. M. Ito, T. Kenmotsu, H. Nakamura, "Extenson of Binary-Collision-Approximation-Based Simulation Applicable to Any Structured Target Material," Jpn. J. Appl. Phys., 50, 01AB03 (2011).

7) S. Saito, A. M. Ito, A. Takayama, T. Kenmotsu, H. Nakamura, "Hybrid Simulation between Molecular Dynamics and Binary Collision Approximation Codes for Hydrogen injection into Carbon Materials," J. Nucl. Mater, doi:10.1016/ j.jnucmat.201012.233, (2011).

8) G. Moliere, Z. Naturforsch., 2A, 133 (1947).

9) H. H. Andersen, J. F. Ziegler, The Stopping and Ranges of Ions in Matter, Vol. 3, Pergamon Press, Oxford, (1977).

10) H. A. Bethe, "Zur Theorie des Durchgangs schneller Korpuskularstrahlen durch Materie," Ann. der Physik, 5, 325-400 (1930).

11) J. Lindhard, M. Scharff, H. E. Schiött, K. Dan. Vidensk. Selsk, Mat. Fys. Medd., 33, No. 14, (1963).

12) Y. Yamamura, Y. Mizuno, "LOW-ENERGY SPUTTERINGS WITH THE MONTE CARLO PROGRAM ACAT," Inst. Plasma Phys., Nagoya Univ., Japan, IPPJ-AM-40, (1985).

13) A. Ito, H. Nakamura, "Erosion of Graphene in Hydrogen Atom Gas,” Jpn. J. Appl. Phys., 47, 4715-4718 (2008).

14) M. Suzuki, "Decomposition formulas of exponential operators and Lie exponentials with some applications to quantum mechanics and statistical physics," J. Math. Phys., 26, 601-612 (1985). 